Research

\title{
In vitro activity of pyronaridine against Plasmodium falciparum and comparative evaluation of anti-malarial drug susceptibility assays Florian Kurth*1,2,3, Peter Pongratz ${ }^{1,2}$, Sabine Bélard ${ }^{1,2,4}$, Benjamin Mordmüller ${ }^{1,2}$, Peter G Kremsner ${ }^{1,2}$ and Michael Ramharter ${ }^{1,5}$
}

\begin{abstract}
Address: ${ }^{1}$ Medical Research Unit, Albert Schweitzer Hospital, Lambaréné, Gabon, ${ }^{2}$ Institute for Tropical Medicine, Department of Parasitology, University of Tübingen, Tübingen, Germany, ${ }^{3}$ Department of Paediatrics, University Hospital Carl Gustav Carus Dresden, Dresden, Germany, ${ }^{4}$ Department of Pediatrics and Adolescent Medicine, University Freiburg, Freiburg, Germany and ${ }^{5}$ Department of Medicine I, Division of Infectious Diseases and Tropical Medicine, Medical University of Vienna, Vienna, Austria

Email: Florian Kurth* - florian.kurth@uniklinikum-dresden.de; Peter Pongratz - PPongratz@gmx.at; Sabine Bélard - sabinebelard@yahoo.de; Benjamin Mordmüller - benjamin.mordmueller@uni-tuebingen.de; Peter G Kremsner - peter.kremsner@uni-tuebingen.de;

Michael Ramharter - michael.ramharter@meduniwien.ac.at

* Corresponding author
\end{abstract}

Published: 23 April 2009

Malaria Journal 2009, 8:79 doi:10.1 186/1475-2875-8-79

This article is available from: http://www.malariajournal.com/content/8/I/79

(c) 2009 Kurth et al; licensee BioMed Central Ltd.

This is an Open Access article distributed under the terms of the Creative Commons Attribution License (http://creativecommons.org/licenses/by/2.0), which permits unrestricted use, distribution, and reproduction in any medium, provided the original work is properly cited.
Received: 18 November 2008
Accepted: 23 April 2009

\begin{abstract}
Background: Pyronaridine, a Mannich base anti-malarial with high efficacy against drug resistant Plasmodium falciparum, is currently evaluated as a fixed dose combination with artesunate for the treatment of uncomplicated malaria. In this study, the in vitro activity of pyronaridine against clinical isolates of $P$. falciparum from Lambaréné, Gabon, was assessed in order to obtain baseline data on its activity prior to its future use in routine therapy. Moreover, follow-up assessment on the in vitro activity of chloroquine, artesunate and quinine was performed.

Methods: In vitro response of field isolates of $P$. falciparum to pyronaridine, chloroquine, artesunate and quinine was assessed using the traditional WHO microtest. In addition, the histidine-rich protein 2 (HRP-2) assay was performed and evaluated for its future implementation for follow-up of drug susceptibility testing.

Results: Pyronaridine exhibited a high in vitro activity against $P$. falciparum, with a geometric mean cut-off concentration of $9.3 \mathrm{nmol} / \mathrm{l}$. Fifty percent effective concentrations were $1.9 \mathrm{nmol} / \mathrm{l}$ and 2.0 $\mathrm{nmol} / \mathrm{l}$ in the WHO microtest and HRP-2 assay, respectively. Results matched closely in vivo findings from a recent clinical trial on pyronaridine-artesunate treatment. One isolate showed diminished sensitivity to artesunate. For chloroquine and quinine resistance levels were comparable to prior studies from Lambaréné. Results from the novel HRP-2 assay corresponded well to those obtained by the WHO microtest.
\end{abstract}

Conclusion: Pyronaridine is highly active in chloroquine-resistant parasites and seems a promising partner drug for artemisinin-based combination therapy in Africa.

\section{Background}

Malaria continues to be a major cause of morbidity and mortality in sub-Saharan Africa, particularly in young children. Early detection and effective chemotherapy remain the cornerstones in its control [1]. The rapid development and spread of anti-malarial drug resistance has 
made surveillance of drug sensitivity a high priority issue. In addition to assessing the activity of common antimalarials against Plasmodium falciparum in routine surveys, the evaluation of new compounds against field isolates is of major importance for drug development.

In Lambaréné, Gabon, routine anti-malarial drug susceptibility monitoring has been performed since 1992 [2-5]. So far the WHO microtest - one of the longest used and best validated assays for the assessment of in vitro drug sensitivity under field conditions - has been used for this purpose [6]. Meanwhile, novel methods in drug susceptibility testing have been developed, such as the histidinerich protein II (HRP-2) assay [7]. This method, based on HRP-2 measurement in an enzyme-linked immunosorbent assay (ELISA), is equally simple to implement, but considerably less labour intensive compared with the WHO microtest. Due to these advantages, the WHO microtest will be replaced for standard drug susceptibility monitoring in Lambaréné in the future by the novel HRP2 assay. Previous findings from laboratory adapted clones and from different geographical regions suggest that results obtained by the HRP-2 assay be comparable with those obtained by the WHO microtest [7].

The anti-malarial agent pyronaridine is a Mannich base derivative of mepacrine, one of the earliest synthetic antimalarials [8]. It is currently evaluated as a fixed dose combination with artesunate for the treatment of uncomplicated falciparum and vivax malaria in adult and paediatric patients [9]. Its anti-plasmodial activity involves interference with the glutathione-dependent detoxification of haem and targeting of $\beta$-haematin formation [10]. Reports from paediatric patients in Africa showed that pyronaridine is effective against chloroquine resistant strains of $P$. falciparum in vivo $[9,11]$, yet data from SouthEast Asia indicate the potential for rapid development of resistance against pyronaridine, when used as monotherapy [12].

The present study aimed to assess the susceptibility of clinical $P$. falciparum field isolates from Lambaréné to pyronaridine in order to obtain baseline data on the activity of this for Africa yet novel anti-malarial drug prior to its widespread use in routine therapy. In addition the study was designed to assess the potential for the novel HRP-2 assay to replace the standard WHO microtest.

\section{Methods}

\section{Study area and patients}

The study was carried out at the Medical Research Unit of the Albert Schweitzer Hospital in Lambaréné, Gabon, in a region of stable, hyperendemic $P$. falciparum malaria transmission $[13,14]$. Patients attending the outpatient clinic between March and October 2006 were asked to participate in the study if they met the following inclusion criteria: P. falciparum monoinfection with 1,000-100,000 asexual parasites per $\mu$ l blood, no schizontaemia, no signs or symptoms of severe malaria, and no history of intake of anti-malarial drugs in the preceding month. Information about age, sex, and duration of fever was collected on a questionnaire. Informed consent was obtained from participants or their legal representatives. The study was approved by the Ethics Committee of the International Foundation for the Albert Schweitzer Hospital in Lambaréné.

\section{In vitro drug sensitivity assays}

Two different methods of drug sensitivity testing were deployed in order to assess the susceptibility of fresh field isolates of $P$. falciparum to pyronaridine, artesunate, chloroquine and quinine. First, the standard World Health Organization in vitro microtest was used similar to previous reports, measuring drug-dependent inhibition of schizont maturation (SMI) within 24 hours by microscopic assessment [2-5]. Briefly, two millilitres of venous blood were mixed with complete parasite culture medium (RPMI 1640, $200 \mu \mathrm{M}$ hypoxanthine, $25 \mathrm{mM}$ Hepes, $0.5 \%$ albumax, $2 \mathrm{mM}$ l-glutamine) to a final concentration of $5 \%$ blood medium mixture (BMM). Ninety-six-well test plates were pre-dosed in ascending quantities of drugs, dosing each plate with all respective drugs for one isolate. Final drug concentrations were 0.5-365.9 nmol/l BMM for pyronaridine (Mr: 910.04), 0.1-85.8 nmol/l BMM for artesunate (Mr: 384.425), 0.8-51.2 $\mu \mathrm{mol} / \mathrm{l}$ blood for chloroquine (Mr: 515.867) and 55-3567 nmol/l BMM for quinine (Mr: 785.06). Artesunate, chloroquine and quinine were dissolved in $70 \%$ ethanol, pyronaridine was resuspended in distilled water. In accordance to the protocols distributed by the World Health Organization drug concentrations for chloroquine are expressed as related to blood due to their considerable accumulation in erythrocytes [6].

Fifty $\mu \mathrm{l}$ BMM were transferred into scheduled wells and incubated at $37.5^{\circ} \mathrm{C}$ in candle jars. After 24 hours parasites were harvested and Giemsa-stained thick blood films were prepared. The number of mature schizonts was microscopically counted against 200 asexual parasites in each well. Tests were considered successful if at least $10 \%$ schizont maturation was observed in the drug-free control-well.

In addition, the HRP-2 assay was performed according to the published standard operational procedures [7]. Two $\mathrm{ml}$ of venous blood were mixed with parasite medium to a concentration of $3 \% \mathrm{BMM}$. Test plates were pre-dosed to the same final concentrations as in the WHO test and incubated for 72 hours at $37^{\circ} \mathrm{C}$ in candle jars. To test for successful in vitro parasite-growth, a thick blood smear of 
one control-well was performed after $26 \mathrm{~h}$. One nontreated $26 \mathrm{~h}$ sample was frozen to calculate background HRP-2 production. Parasite culture was judged successful if at least $10 \%$ parasites matured to schizonts at the 26 hour time point. After $72 \mathrm{~h}$ plates were freeze-thawed twice. Parasite growth, calculated from HRP-2 levels, was measured with an enzyme linked immunosorbent assay at an absorbent maximum of $450 \mathrm{~nm}$.

\section{Statistical analysis}

Non-linear regression analysis with 4-parameter fits of log-concentration/response curves was used to determine individual inhibitory concentrations of the respective isolates. All regressions were checked manually. Cut off concentrations were calculated as geometric mean of the lowest individual concentrations with no mature schizont among 200 parasites in the WHO microtest. Nonparametric analysis was used for concentration data that was not normally distributed. A two-tailed Mann-Whitney-U-Test was performed in order to test for difference between the two drug sensitivity assays. All tests were performed at a two sided significance level of $\alpha=0.05$.

\section{Results}

Ninety-five outpatients attending the Albert Schweitzer Hospital were included in this study. The patients' median age was three years, ranging from three month to 18 years, and 55\% were female. Median asexual parasitaemia at presentation was 36,500 per $\mu$ l blood. In the schizont maturation microtest 32,36, 34, and 32 out of 95 isolates yielded valid results for pyronaridine, chloroquine, artesunate and quinine, respectively. Twenty-five isolates fulfilled the criteria for successful parasite-culture in the HRP-2 assay and 15, 16, 12 and 14 were successfully employed for drug sensitivity testing, respectively. Posthoc analysis of the unexpected low success rate of cultivation, especially in the HRP-2 assay, revealed that poor growth was associated with one lot of the commercially acquired medium. Those isolates were identified and excluded from the analysis. Table 1 shows 50 percent, 99 percent and cut-off concentrations of the tested drugs as obtained by the WHO microtest and HRP-2 assay.

\section{Pyronaridine}

Pyronaridine inhibited in vitro growth and schizont maturation in the WHO assay at a geometric mean cut-off concentration of $9.3 \mathrm{nmol} / \mathrm{l} \mathrm{BMM}$. The respective geometric mean $\mathrm{EC}_{99}$ concentration in the HRP-2 assay was 9.0 $\mathrm{nmol} / \mathrm{l}$. In one isolate schizont maturation was observed at a pyronaridine concentration of $122 \mathrm{nmol} / \mathrm{l} \mathrm{BMM}$, resulting in a cut-off concentration of $366 \mathrm{nmol} / \mathrm{l} \mathrm{BMM}$. Further two isolates showed cut-off concentrations of 122 $\mathrm{nmol} / \mathrm{l}$. Geometric mean $\mathrm{EC}_{50}$ values were 1.9 and 2.0 $\mathrm{nmol} / \mathrm{l} \mathrm{BMM}$ for the WHO microtest and the HRP-2 assay, respectively.

\section{Chloroquine}

The geometric mean cut-off concentration for chloroquine was $21.7 \mu \mathrm{mol} / \mathrm{l}$ blood in the WHO assay, 19.2 $\mu \mathrm{mol} / \mathrm{l}$ blood was the respective $\mathrm{EC}_{99}$ value in the HRP-2 assay. The most sensitive isolate showed a cut-off concentration of $3.2 \mu \mathrm{mol} / 1$ blood, all other isolated exhibited cut-off concentrations of $6.4 \mu \mathrm{mol} / \mathrm{l}$ blood or higher. In four parasite cultures, microscopic assessment revealed schizont maturation up to the highest concentration of $51.3 \mu \mathrm{mol} / \mathrm{l}$ blood. The 50 percent effective concentrations were $7.1 \mu \mathrm{mol} / \mathrm{l}$ blood in the WHO microtest and $5.5 \mu \mathrm{mol} / \mathrm{l}$ in the HRP-2 assay, respectively.

\section{Artesunate}

Fifty percent effective concentrations were $2.1 \mathrm{nmol} / \mathrm{l}$ BMM in the microtest and $2.3 \mathrm{nmol} / \mathrm{l} \mathrm{BMM}$ in the HRP-2 assay for artesunate. Two isolates exhibited schizont maturation at $28.6 \mathrm{nmol} / \mathrm{l} \mathrm{BMM}$, yielding cut-off concentrations of $85 \mathrm{nmol} / \mathrm{l} \mathrm{BMM}$. In one isolate in vitro schizont maturation was not inhibited by the highest artesunate concentration of $85 \mathrm{nmol} / \mathrm{l} \mathrm{BMM}$. The same isolate exhibited good sensitivity to pyronaridine and to quinine. The geometric mean cut-off concentration was $9.2 \mathrm{nmol} / \mathrm{l}$

Table I: $\mathrm{EC}_{50}, \mathrm{EC}_{99}$ and cut-off concentrations of pyronaridine, chloroquine, artesunate and quinine in WHO microtest and HRP-2 assay

\begin{tabular}{|c|c|c|c|c|c|c|}
\hline & \multicolumn{3}{|c|}{ WHO Microtest } & \multicolumn{3}{|c|}{ HRP-2 assay } \\
\hline & $N$ & $\mathrm{EC}_{50}(95 \% \mathrm{Cl})$ & Cut-off concentration $(95 \% \mathrm{Cl})$ & $N$ & $\mathrm{EC}_{50}(95 \% \mathrm{Cl})$ & $\mathrm{EC}_{99}(95 \% \mathrm{Cl})$ \\
\hline Pyronaridine & 32 & $\begin{array}{c}1.87 \\
(1.40-2.48)\end{array}$ & $\begin{array}{c}9.32 \\
(5.2-13.9)\end{array}$ & 15 & $\begin{array}{c}2.03 \\
(1.57-2.63)\end{array}$ & $\begin{array}{c}9.03 \\
(6.42-12.69)\end{array}$ \\
\hline Chloroquine & 36 & $\begin{array}{c}7.1 \\
(5.2-9.6)\end{array}$ & $\begin{array}{c}21.7 \\
(15.4-30.6)\end{array}$ & 16 & $\begin{array}{c}5.5 \\
(4.4-6.9)\end{array}$ & $\begin{array}{c}19.2 \\
(15.3-24.2)\end{array}$ \\
\hline Artesunate & 34 & $\begin{array}{c}2.08 \\
(1.48-2.92)\end{array}$ & $\begin{array}{c}9.24 \\
(6.53-13.06)\end{array}$ & 12 & $\begin{array}{c}2.25 \\
(1.70-2.99)\end{array}$ & $\begin{array}{c}15.24 \\
(10.45-22.24)\end{array}$ \\
\hline Quinine & 32 & $\begin{array}{c}272 \\
(210-353)\end{array}$ & $\begin{array}{c}873 \\
(723-1053)\end{array}$ & 14 & $\begin{array}{c}204 \\
(167-250)\end{array}$ & $\begin{array}{c}662 \\
(56 I-78 I)\end{array}$ \\
\hline
\end{tabular}

All values are in nmol/l BMM except for chloroquine ( $\mu \mathrm{mol} / \mathrm{l}$ blood),

Means and $95 \%$ confidence intervals are depicted as antilog of arithmetic mean of log-transformed data 
BMM. The HRP-2 assay showed a mean $\mathrm{EC}_{99}$ level of 15.2 nmol/l BMM.

\section{Quinine}

All isolates were susceptible to quinine. Highest cut-off concentrations were at $1783 \mathrm{nmol} / \mathrm{l} \mathrm{BMM}$, thus well below the threshold of resistance (5120 nmol/l BMM). The geometric mean cut-off concentration was $873 \mathrm{nmol} /$ $\mathrm{l}$, the $\mathrm{EC}_{99}$ in the HRP-2 assay was $662 \mathrm{nmol} / \mathrm{l} \mathrm{BMM}$. The $\mathrm{EC}_{50}$ values in the WHO microtest and in the HRP-2 assay were 272 and $204 \mathrm{nmol} / \mathrm{l} \mathrm{BMM}$, respectively.

\section{Activity correlation of the tested drugs}

Correlation analysis of $\mathrm{EC}_{50}$ values of the individual isolates was performed in order to assess the relationship between sensitivity of parasites to pyronaridine, chloroquine, artesunate and quinine (Table 2). Significant correlation was found between in vitro activities of the antimalarials quinine and chloroquine $(\mathrm{r}=0.50, \mathrm{p}<0.005)$. Interestingly, the activity of pyronaridine was correlated with artesunate $(\mathrm{r}=0.84 \mathrm{p}<0.0001)$, but not with chloroquine or quinine.

\section{Comparison of HRP-2 assay and WHO microtest}

No statistically significant differences between the $\mathrm{EC}_{50}$ values from the two drug sensitivity assays were found in U-test of pooled data of the individual drugs $(p=0.82$ for pyronaridine, 0.72 for artesunate, 0.11 for chloroquine and 0.20 for quinine). Differences in geometric means of $\mathrm{EC}_{50}$ values were largest for chloroquine $(7.1 \mu \mathrm{mol} / \mathrm{l}, 95 \%$ CI 5.2-9.6 in the WHO microtest versus $5.5 \mu \mathrm{mol} / 195 \%$ CI: 4.4-6.9 in the HRP-2 assay, $\mathrm{p}=0.11$ ) and quinine (272 nmol/l 95\% CI: 210-353 versus $204 \mathrm{nmol} / 1$ 95\%CI $167-250$, respectively. $\mathrm{p}=0.20$ ). Figure 1 shows the distribution of $\mathrm{EC}_{50}$ values for the respective drugs.

Cut-off concentrations as determined by the WHO microtest correlate closely to the equivalent $\mathrm{EC}_{99}$ values of the HRP-2 assay (Table 1). Significantly more isolates yielded

Table 2: Activity correlation between pyronaridine, chloroquine, artesunate and quinine in fresh Plasmodium falciparum isolates

\begin{tabular}{llccc}
\hline & & Pyronaridine & Chloroquine & Artesunate \\
\hline Chloroquine & $\mathrm{R}^{2}$ & $\mathbf{0 . 1 3}$ & - & - \\
& $\mathrm{P}$ & 0.52 & & \\
\multirow{5}{*}{ Artesunate } & $\mathrm{N}$ & 30 & & \\
& $\mathrm{R}^{2}$ & $\mathbf{0 . 8 4}$ & $-\mathbf{0 . 0 7}$ & - \\
& $\mathrm{P}$ & $<.0001^{*}$ & 0.69 & \\
Quinine & $\mathrm{N}$ & 29 & 30 & \\
& $\mathrm{R}^{2}$ & $\mathbf{0 . 0 6}$ & $\mathbf{0 . 5 0}$ & $\mathbf{0 . 0 2}$ \\
& $\mathrm{P}$ & 0.74 & $0.0037^{*}$ & 0.93 \\
& $\mathrm{~N}$ & 30 & 31 & 30 \\
\hline
\end{tabular}

$\mathrm{R}^{2}$ : correlation coefficient in pairwise correlation analysis

* statistically significant valid results in the microtest (36\%) than in the HRP-2 based assay $(15 \%)(\mathrm{p}<0.001)$.

\section{Discussion}

Pyronaridine exhibited a high level of in vitro activity against isolates of $P$. falciparum from infected Gabonese subjects in this study. Fifty percent effective concentrations were 2.0 and $1.9 \mathrm{nmol} / \mathrm{l}$, which is consistent with previously published data from studies with laboratory strains and field isolates obtained by a different drug sensitivity assay (isotopic hypoxanthine assay) $[15,16]$.

Median pyronaridine concentrations of $9.3 \mathrm{nmol} / \mathrm{l}\left(\mathrm{EC}_{99}\right.$ in HRP-2 assay) and $9.0 \mathrm{nmol} / \mathrm{l}$ (cut-off concentration in WHO microtest) inhibited growth of $P$. falciparum in vitro. In a recent pharmacokinetic assessment of a fixed-dose pyronaridine artesunate combination in African children, the mean maximal plasma concentrations of pyronaridine were $115 \mathrm{nmol} / \mathrm{l}$ in patients [9]. Earliest reappearances of parasites in peripheral blood in this study were observed on day 21 (one patient) and day 28 (three patients) after treatment. Interestingly, the patient with parasite reappearance on day 21 exhibited a pyronaridine plasma level of $9.9 \mathrm{nmol} / \mathrm{l}$ at that time. In polynomial regression analysis of pooled plasma levels of all patients, mean pyronaridine blood concentrations fell below levels of $9 \mathrm{nmol} / \mathrm{l}$ on day 22. First reappearances in vivo therefore occurred only after plasma levels fell below concentrations similar to cut-off concentrations obtained in this in vitro study.

Although the extrapolation of in vitro drug sensitivity assessments to in vivo data should always be cautious [17], in vitro results of the present study matched closely clinical findings of this clinical trial on pyronaridine-artesunate combination therapy.

The relationship between in vitro activity of pyronaridine and chloroquine against $P$. falciparum has been discussed controversially in previous reports and contradictory conclusions concerning cross-resistance have been drawn from studies, which found a significant correlation between pyronaridine and chloroquine in vitro activity and differences in activity of pyronaridine against chloroquine resistant and sensitive strains $[15,16,18,19]$. In the present study, in vitro response to chloroquine was poor in all tested isolates, yet parasites proved to be highly susceptible to pyronaridine. There was no statistically significant correlation of the in vitro activities of the two drugs against $P$. falciparum. These in vitro findings and the recently reported high cure rates of three days pyronaridine-artesunate combination therapy provide convincing evidence against a clinically significant cross resistance of pyronaridine and chloroquine in vivo. The results underline the usefulness of pyronaridine for the treatment of patients in 

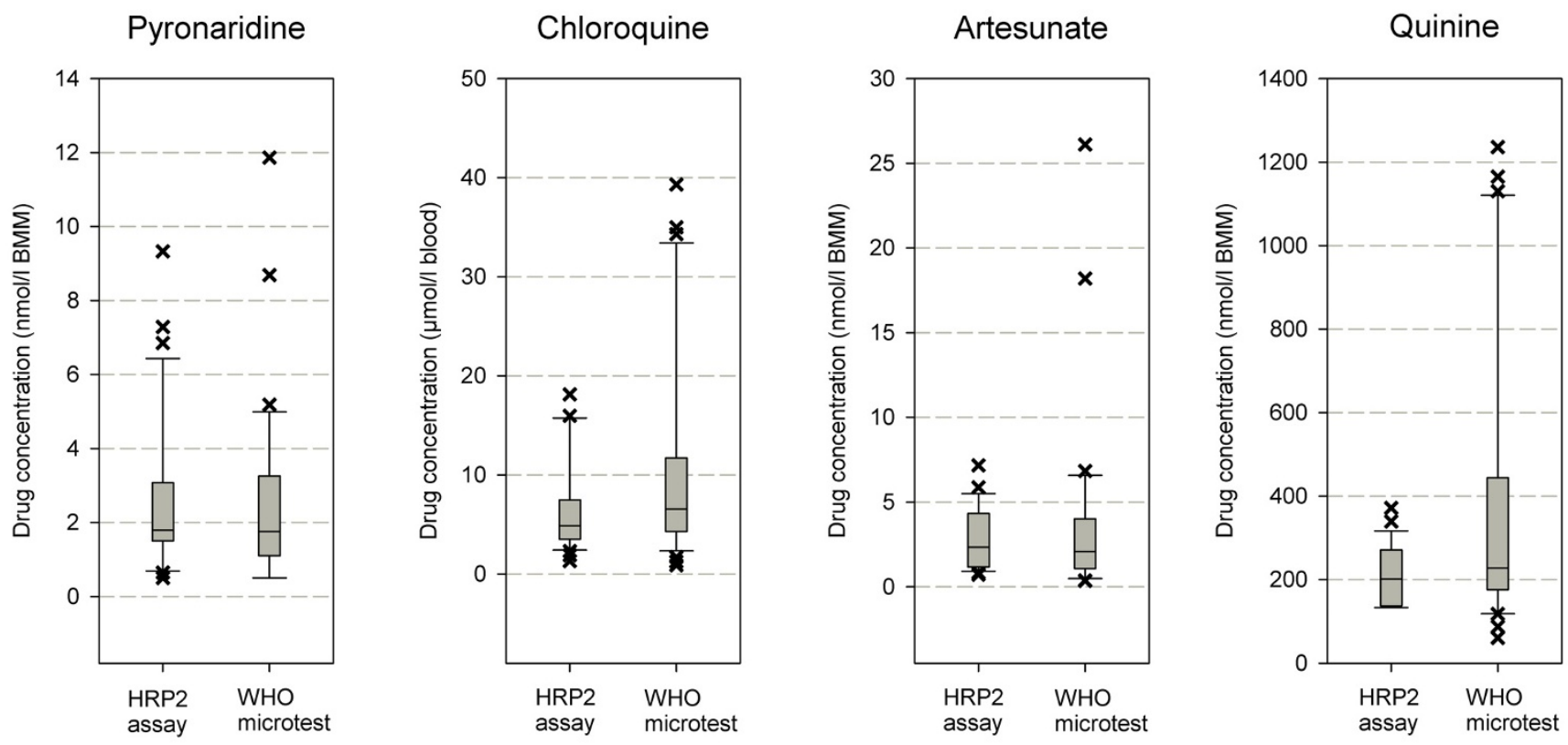

Figure I

Box plots of pyronaridine, chloroquine, artesunate and quinine $\mathrm{EC}_{50}$ in HRP-2 assay and WHO microtest.

regions affected by $P$. falciparum resistant to chloroquine $[9,11]$.

Since the first in vitro drug susceptibility assessment in Lambaréné in 1992 [2], high levels of resistance against chloroquine have been observed in vitro and in vivo [3-5]. Similar to previous studies in vitro response to chloroquine was poor in this study. $\mathrm{EC}_{50}$ levels were comparable to the last assessment in 2002 [5], despite a decrease in drug pressure due to a change of national treatment policies to artemisinin-based combination therapy in 2003. Artesunate was highly active against $P$. falciparum in the present assessment. $\mathrm{EC}_{50}$ levels were similar to recent in vitro and in vivo findings at the study site and in neighbouring countries [20-22]. One isolate showed a diminished sensitivity to artesunate with schizont maturation up to the highest concentration of $85.8 \mathrm{nmol} / \mathrm{l}$ and a corresponding $\mathrm{EC}_{50}$ level of $19.9 \mathrm{nmol} / \mathrm{l}$. Another isolate had a comparably high $\mathrm{EC}_{50}$ value of $10.1 \mathrm{nmol} / \mathrm{l}$ and a respective cut-off concentration in the WHO microtest of 85.8 $\mathrm{nmol} / \mathrm{l}$. These findings support recent in vitro susceptibility assessments by Cojean et al [23], reporting on 6 out of 397 African $P$. falciparum isolates with dihydroartemisinin $\mathrm{EC}_{50}$ levels above $10 \mathrm{nmol} / \mathrm{l}$ and a maximum $\mathrm{EC}_{50}$ of 31.8 $\mathrm{nmol} / \mathrm{l}$ in one isolate. Especially in the light of increasing availability and use of artemisinins in many parts of Africa, the importance of close surveillance for susceptibility as well as strict deployment of artemisinins exclusively in combination therapy with effective partner drugs cannot be overemphasized.

As in previous reports from Lambaréné, susceptibility to quinine was high in this study. Mean $\mathrm{EC}_{50}$ (204 and 272 nmol/l in HRP-2 assay and WHO microtest, respectively) were comparable to results obtained in 2002 (286 nmol/ 1) [5]. Despite its widespread use, quinine remains a highly effective anti-malarial in Gabon, especially as drug of choice for parenteral treatment of hospitalized patients.

Comparative assessment of the HRP-2 assay and WHO microtest was performed in order to establish baseline data for the use of the ELISA based assay in Lambaréné in the future. Results obtained by the HRP-2 assay closely paralleled those obtained by the WHO microtest despite methodological problems in this study due to a deficient batch of culture medium. Considering its reduced labourintensity and good reproducibility the HRP-2 assay can, therefore, be recommended for follow up of anti-malarial drug susceptibility testing and will replace the WHO microtest in future assessments at our study site.

\section{Conclusion}

Artesunate, chloroquine and quinine in vitro drug activity was similar, compared to data from earlier in vitro assessments, and results of the HRP-2 assay were comparable to those obtained by the traditional WHO microtest. 
The study demonstrated high anti-malarial activity of pyronaridine against fresh field isolates of $P$. falciparum and corresponded well to recent findings of pyronaridine antimalarial activity in vivo. Pyronaridine is recommended for further clinical development in combination therapy and continued in vitro drug activity monitoring.

\section{Competing interests}

The authors declare that they have no competing interests.

\section{Authors' contributions}

FK contributed to the conception and design of the study, performed parasite cultivation and microscopic assessment, analysed the data and wrote the manuscript. PP performed parasite cultivation and HRP-2 immunoassay. SB gathered field isolates from patients and performed parasite cultivation. BM contributed to performance of immunoassay and analysis of data, PGK revised the manuscript and supervised the research group, MR conceived and designed the study, contributed to analysis of data, performed second reading for microscopic assessments in WHO microtest and drafted and revised the manuscript. All authors read and approved the final version of the manuscript.

\section{Acknowledgements}

We thankfully acknowledge the participations of our patients in the Albert Schweitzer Hospital in Lambaréné. This study was supported by a grant of the independent Karl Landsteiner Gesellschaft, Austria.

\section{References}

I. Kremsner PG, Krishna S: Antimalarial combinations. Lancet 2004, 364:285-294.

2. Winkler S, Brandts C, Wernsdorfer WH, Graninger W, Bienzle U, Kremsner PG: Drug sensitivity of Plasmodium falciparum in Gabon. Activity correlations between various antimalarials. Trop Med Parasitol 1994, 45:214-218.

3. Philipps J, Radloff PD, Wernsdorfer W, Kremsner PG: Follow-up of the susceptibility of Plasmodium falciparum to antimalarials in Gabon. Am J Trop Med Hyg 1998, 58:6।2-618.

4. Borrmann S, Binder RK, Adegnika AA, Missinou MA, Issifou S, Ramharter M, Wernsdorfer WH, Kremsner PG: Reassessment of the resistance of Plasmodium falciparum to chloroquine in Gabon: implications for the validity of tests in vitro vs. in vivo. Trans $R$ Soc Trop Med Hyg 2002, 96:660-663.

5. Ramharter $M$, Wernsdorfer WH, Kremsner $P G$ : In vitro activity of quinolines against Plasmodium falciparum in Gabon. Acta Trop 2004, 90:55-60.

6. In vitro test (mark II) for the assessment of the response of Plasmodium falciparum to chloroquine, mefloquine, quinine, sulfadoxine/pyrimethamine and amodiaquine. WHO document CTD/MAL/97.20 Rev 200 I [http://www.who.int/ drugresistance/malaria/en/markiii.pdf]

7. Noedl H, Wernsdorfer WH, Miller RS, Wongsrichanalai C: Histidine-rich protein II: a novel approach to malaria drug sensitivity testing. Antimicrob Agents Chemother 2002, 46: I658-I664.

8. Chang $C$, Lin-Hua T, Jantanavivat C: Studies on a new antimalarial compound: pyronaridine. Trans $R$ Soc Trop Med Hyg 1992 86:7-10.

9. Ramharter M, Kurth F, Schreier AC, Nemeth J, Glasenapp IV, Belard S, Schlie M, Kammer J, Koumba PK, Cisse B, Mordmuller B, Lell B, Issifou S, Oeuvray C, Fleckenstein L, Kremsner PG: Fixed-dose pyronaridine-artesunate combination for treatment of uncomplicated falciparum malaria in pediatric patients in Gabon. J Infect Dis 2008, 198:911-919.
10. Auparakkitanon S, Chapoomram S, Kuaha K, Chirachariyavej T, Wilairat P: Targeting of hematin by the antimalarial pyronaridine. Antimicrob Agents Chemother 2006, 50:21 97-2200.

II. Ringwald P, Bickii J, Basco LK: Efficacy of oral pyronaridine for the treatment of acute uncomplicated falciparum malaria in African children. Clin Infect Dis 1998, 26:946-953.

12. Yang HL, Liu DQ, Yang YM, Huang KG, Dong Y, Yang PF, Liao MZ, Zhang CY: In vitro sensitivity of Plasmodium falciparum to eight antimalarials in China-Myanmar and China-Lao PDR border areas. Southeast Asian J Trop Med Public Health 1997, 28:460-464.

13. Ramharter M, Adegnika AA, Agnandji ST, Matsiegui PB, Grobusch MP, Winkler S, Graninger W, Krishna S, Yazdanbakhsh M, Mordmüller B, Lell B, Missinou MA, Mavoungou E, Issifou S, Kremsner PG: History and perspectives of medical research at the Albert Schweitzer Hospital in Lambaréné, Gabon. Wien Klin Wochenschr 2007, I | 9:8-12

14. Wildling E, Winkler S, Kremsner PG, Brandts C, Jenne L, Wernsdorfer WH: Malaria epidemiology in the province of Moyen Ogoov, Gabon. Trop Med Parasitol 1995, 46:77-82.

15. Pradines B, Mabika Mamfoumbi M, Parzy D, Owono Medang M, Lebeau C, Mourou Mbina JR, Doury JC, Kombila M: In vitro susceptibility of African isolates of Plasmodium falciparum from Gabon to pyronaridine. Am J Trop Med Hyg 1999, 60:105-108.

16. Vivas L, Rattray L, Stewart L, Bongard E, Robinson BL, Peters W, Croft SL: Anti-malarial efficacy of pyronaridine and artesunate in combination in vitro and in vivo. Acta Trop 2008, 105:222-228.

17. Ekland EH, Fidock DA: In vitro evaluations of antimalarial drugs and their relevance to clinical outcomes. Int J Parasitol 2008, 38:743-747.

18. Pradines B, Tall A, Parzy D, Spiegel A, Fusai T, Hienne R, Trape JF, Doury JC: In-vitro activity of pyronaridine and amodiaquine against African isolates (Senegal) of Plasmodium falciparum in comparison with standard antimalarial agents. J Antimicrob Chemother 1998, 42:333-339.

19. Elueze El, Croft SL, Warhurst DC: Activity of pyronaridine and mepacrine against twelve strains of Plasmodium falciparum in vitro. J Antimicrob Chemother 1996, 37:5 I I-518.

20. Pradines B, Hovette $P$, Fusai T, Atanda HL, Baret E, Cheval P, Mosnier J, Callec A, Cren J, Amalvict R, Gardair JP, Rogier C: Prevalence of in vitro resistance to eleven standard or new antimalarial drugs among Plasmodium falciparum isolates from PointeNoire, Republic of the Congo. J Clin Microbiol 2006, 44:2404-2408.

21. Ramharter M, Kurth FM, Belard S, Bouyou-Akotet MK, Mamfoumbi MM, Agnandji ST, Missinou MA, Adegnika AA, Issifou S, Cambon N, Heidecker JL, Kombila M, Kremsner PG: Pharmacokinetics of two paediatric artesunate mefloquine drug formulations in the treatment of uncomplicated falciparum malaria in Gabon. Antimicrob Chemother 2007, 60:1091-1096.

22. Ramharter M, Noed H, Thimasarn K, Wiedermann G, Wernsdorfer $\mathrm{G}$, Wernsdorfer WH: In vitro activity of tafenoquine alone and in combination with artemisinin against Plasmodium falciparum. Am J Trop Med Hyg 2002, 67:39-43.

23. Cojean S, Hubert V, Le Bras J, Durand R: Resistance to dihydroartemisinin. Emerg Infect Dis 2006, I 2:I798-I799.

Publish with Bio Med Central and every scientist can read your work free of charge

"BioMed Central will be the most significant development for disseminating the results of biomedical research in our lifetime. "

Sir Paul Nurse, Cancer Research UK

Your research papers will be:

- available free of charge to the entire biomedical community

- peer reviewed and published immediately upon acceptance

- cited in PubMed and archived on PubMed Central

- yours - you keep the copyright

BioMedcentral 